Nouvelles perspectives en sciences sociales

Revue internationale de systémique complexe et d'études relationnelles

\title{
Dons cachés, normes matrimoniales et règles d'héritage dans les échanges au sein des ménages à Lubumbashi
}

\section{Olivier Kahola}

Volume 9, numéro 2, mai 2014

Sur le thème de l'échange

URI : https://id.erudit.org/iderudit/1025974ar

DOI : https://doi.org/10.7202/1025974ar

Aller au sommaire du numéro

\section{Éditeur(s)}

Prise de parole

ISSN

1712-8307 (imprimé)

1918-7475 (numérique)

Découvrir la revue

Citer cet article

Kahola, O. (2014). Dons cachés, normes matrimoniales et règles d'héritage dans les échanges au sein des ménages à Lubumbashi. Nouvelles perspectives en sciences sociales, 9(2), 159-185. https://doi.org/10.7202/1025974ar
Résumé de l'article

Régulièrement, nous entendons que les Africains sont solidaires. Ce discours encensant cache bien d'autres formes d'échanges entre les parents, notamment les aides clandestines envers les familles d'origine des conjoints. Le plus souvent, on se contente des réalités présentées sur la sphère publique comme le transfert aux parents des biens matériels et de l'argent pour essentialiser la solidarité dite africaine. Un double jeu social entre les solidarités officielles et ostentatoires (les hébergements) et les solidarités officieuses et cachées (aides clandestines) détermine les recompositions familiales. En même temps, les aides clandestines soulèvent la question des limites entre l'immoralité et la moralité dans l'exercice des solidarités. Cet article poursuit deux objectifs : décrire les coulisses des pratiques de la solidarité dans les ménages et établir une relation entre les aides clandestines et les normes matrimoniales. 


\title{
Dons cachés, normes matrimoniales et règles d'héritage dans les échanges au sein des ménages à Lubumbashi ${ }^{1}$
}

\author{
Olivier Kahola \\ Université de Lubumbashi (RDC)
}

\section{Introduction}

T e transfert des parents et la circulation des dons sont les Laspects visibles de l'extériorisation des solidarités familiales. Cependant, il n'y a pas que des solidarités officielles et ostentatoires (accueil des parents, aides financières ou matérielles aux parents, etc.) mais aussi des solidarités cachées dirigées vers les familles de conjoints. Ces aides cachées relèvent de l'informel et assurent ce que Hugues Leclercq appelle une «survie précaire des proches parents ${ }^{2} »$.

Cet article essaie de montrer que l'échange des dons cachés ou officiels n'est pas motivé que par «l'engrenage de la dette communautaire et de la créance ${ }^{3}$ ", qu'il l'est aussi, par d'autres

$\overline{1}$ Deuxième grande ville de la République Démocratique du Congo. Ces habitants sont appelés "Lushois ".

2 Hugues Leclercq, "L'Économie populaire informelle de Kinshasa. Approche macro-économique ", Zaïre-Afrique, n 271, 1993, p. 19.

3 Alain Marie, "Avatars de la dette communautaire. Crise des solidarités, sorcellerie et procès d'individualisation (itinéraires abidjanais)», Alain Marie (dir.), L'Afrique des individus, Paris, Karthala, 1997, p. 251. 
déterminants comme la valorisation de soi, les règles d'héritage et les normes matrimoniales. J'analyse l'introspection des interfaces entre la scène domestique et les coulisses pour discuter des logiques reproductrices de l'échange caché dans les ménages.

Quatre points font l'ossature de cet article. Le premier point présente quelques caractéristiques socioéconomiques des informateurs. Le deuxième point aborde les situations d'échange des dons cachés. Il dégage les diverses motivations des dons cachés. Le troisième point discute des logiques matrimoniales dans l'exercice des dons cachés. Il montre que les échanges souterrains sont orientés par les normes prescriptives de genre. Le quatrième point, enfin, dégage les règles d'héritage comme l'un des facteurs des échanges cachés.

\subsection{Problématique}

Au cours de son étude sur les ménages, Pierre Petit constate que la crise économique condamne plusieurs Lushois à se tourner vers l'informel ${ }^{4}$. Gauthier de Villers le désigne comme des formes atypiques (non conformes à des modèles culturels), composites (produites par hybridation, métissage de formes issues des matrices culturelles hétérogènes) et ambiguës, polysémiques (se référant à des codes culturels différents $)^{5}$. L'informel est dynamique dans les échanges entre les conjoints et leur famille d'origine. À Lubumbashi, les diverses aides entre les apparentés sont échangées dans l'informel. Cette réalité sociale rencontre l'observation de Jacques Godbout et Johanne Charbonneau selon laquelle une " grande partie de l'aide qui circule entre les adultes continue à être dispensée de façon informelle, notamment par la famille et le réseau de parenté6 ${ }^{6}$. L'informel qu'évoquent ces auteurs diffère des dons cachés que j’observe dans les ménages lushois. Ces dons s'exercent à l'insu d'un des conjoints et mettent

\footnotetext{
$4 \quad$ Pierre Petit (dir.), Ménages de Lubumbashi entre précarité et recomposition, Paris, L'Harmattan, 2003.

5 Gauthier de Villers, "Propositions introductives ", Phénomènes informels et dynamiques culturelles en Afrique, Cahiers Africains, $\mathrm{n}^{\text {os }}$ 19-20, 1996, p. 8-9.

6 Jacques Godbout et Johanne Charbonneau, Circulation du don dans la parenté, une roue qui tourne, Montréal, INRS-Urbanisation, 1996, p.51.
} 
en relation deux ménages distincts économiquement et distants géographiquement.

Pour comprendre les modalités d'exercice des dons cachés, je m’appuie sur les théories de don ${ }^{7}$, de solidarité communautaire ${ }^{8}$. J'entends dépasser le paradigme des obligations sociales du contre-don. Le don est en « apparence libre et désintéressé, mais en réalité, il est contraint et intéressé ${ }^{~}$ ", car " l'arrière-plan de l'échange des dons et des contre-dons est la dette ${ }^{10} \ldots$ ". En analysant les pratiques de solidarités entre les apparentés, il n'y a pas que les sociales de contre-don qui contraignent à l'échange. Je constate que certains conjoints s'engagent dans les dons cachés tantôt pour leur valorisation, tantôt par le poids des normes matrimoniales et des règles d'héritage. À Lubumbashi, les rapports de genre sont prescriptifs. L'homme est perçu comme supérieur à la femme. L'apport de la femme est souvent sousestimé. Ainsi, il revient au mari de prendre totalement en charge l'épouse. Il importe qu'il se montre généreux et attentionné envers sa belle-famille, qu'il ne compte pas sur la participation financière de l'épouse. "Que son salaire soit nettement plus élevé que celui de sa femme ou non, il doit assumer les principales charges financières dans la famille ${ }^{11} »$. Aussi, pour la valorisation de soi, l'homme doit assister ses proches parents. Ces aides sont une manifestation de son indépendance. Une preuve qu'il n'est

$7 \quad$ Alain Caillé, Dé-penser l'économique, Paris, La Découverte/Mauss, 2005; Marcel Mauss, Sociologie et anthropologie, Paris, Presses universitaires de France, coll. "Quadrige ", 1985.

8 Jacky Bouju, «La Sociabilité urbaine et la crise en Afrique de l'ouest : entre les défaillances, de la solidarité familiale et la concurrence cérémonielle, on se cherche! ", dans Georges Ravis-Giordani (dir.), Amitiés. Anthropologie et histoire, Aix-en-Provence, Publications de l'Université de Provence, 1999, p. 325-353; Alain Marie, "Avatars de la dette communautaire. Crise des solidarités, sorcellerie et procès d'individualisation (itinéraires abidjanais)», op. cit., p. 249-328.

9 Marcel Mauss, op. cit., p. 194.

10 Alain Marie, "Une anthropo-logique communautaire à l'épreuve de la mondialisation. De la relation de dette à la lutte sociale (l'exemple ivoirien)", Cahiers d'études Africaines, no 166, 2002/2, p. 210.

11 Sami Tchak, La Sexualité féminine en Afrique, Paris, L'Harmattan, 1999, p. 168. 
pas dominé par la femme. Pour des raisons d'honneur et de secret familial, certains hommes aident leurs parents en cachette. Ils s'arrangent pour que les épouses ne soient pas informées de leurs actions. Dans cette même logique, l'épouse ne doit pas investir son argent dans le ménage. Il ne lui revient pas de supporter le mari. Une épouse qui dépense son argent dans son ménage est traitée de pumbafu (imbécile) par ses proches parents. En cas de divorce ou de décès, elle sera dépouillée du patrimoine matériel accumulé par le ménage. À l'inverse, son argent doit lui profiter ainsi qu'à sa famille d'origine. Le mari se contente d'un regard, assorti de quelques suggestions à la participation au budget ménager; mais il n'est pas un ayant droit; son argent doit être affecté dans le ménage, tout en ayant des retombées dans la belle-famille. C'est de cette manière que l'épouse est valorisée par ses parents. Si le mari ne se montre pas généreux envers la bellefamille, l'épouse, pour sa valorisation sociale, recourt aux dons cachés.

En même temps, les aides envers les familles de conjoints sont orientées par une logique de l'équivalence ${ }^{12}$, c'est-à-dire que chaque conjoint assiste ses parents presque dans la même proportion que son partenaire. Quand l'un des époux estime être marginalisé dans la redistribution des dons, il développe les solidarités cachées.

Face à ces réalités socioculturelles, les aides cachées ne seraientelles pas justifiées par des normes matrimoniales qui exigent que le mari assume la charge familiale tout en conférant à l'épouse un droit sur la progéniture et le travail domestique ? Ces solidarités cachées ne pourraient-elles pas être mises en relation avec les règles d'héritage dans une société où les rapports de genre sont prescriptifs ?

\section{Méthodologie et constitution de l'échantillon}

Je ne suis pas étranger aux réalités de ménage à Lubumbashi. Mon immersion "permet le croisement de divers points de vue

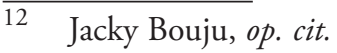


sur l'objet, éclaire la complexité des pratiques, en révèle l'épaisseur $^{13}$ ». Cependant, je devais me " mettre en état de vigilance critique pour être prêt, si nécessaire, à réviser ou à abandonner des concepts que le chercheur considère comme analytiquement fondés ${ }^{14}$ ». Pour pénétrer les réalités domestiques, j'ai combiné les approches qualitative et quantitative dans ma collecte des données. Ce choix méthodologique est légitimé par la poursuite de ce que Jean-Pierre Olivier de Sardan appelle " la plausibilité $^{15}$ ». Mon ethnographie a été appuyée par le questionnaire, les entretiens et les observations directes.

Cette étude est circonscrite à Lubumbashi. Ce choix est justifié par des raisons théorique et pratique. Théorique, car la ville de Lubumbashi est diverse sur le plan culturel, avec un mélange de valeurs occidentales et locales; en outre, bon nombre d'études ont été menées dans cette ville et donnent accès à plusieurs analyses. Pratique, car depuis 2000 je mène des enquêtes ethnographiques dans cette ville; ma connaissance du lieu m'a permis de surmonter certaines difficultés de terrain.

Pour collecter les données, je me suis proposé d'enquêter sur septante ménages compte tenu des moyens financiers et humains à ma disposition. Mes entretiens ont couvert une période de deux mois. Pour connaître le nombre de ménages à enquêter par quartier, j'ai appliqué la règle de trois :

$$
X=\frac{\text { Population du quartier } 2001 \times 70 \text { (Nombre de ménages dansl'échantillon) }}{1180357 \text { (Population de la ville en 2001) }}
$$

Mon échantillon est bâti selon la méthode de quotas. Il respecte le poids démographique de la population dans les différents quartiers. J'ai capitalisé sur les acquis des enquêtes conduites par l’Observatoire dans les ménages en 2008. Les résultats de ces

13 Stéphane Beaud et Florence Weber, Guide de l'enquête de terrain, Paris, La Découverte, 1998, p. 9-10.

14 Maurice Godelier, Les Métamorphoses de la parenté, Paris, Fayard, 2004, p. 33.

15 Jean-Pierre Olivier de Sardan, La Rigueur du qualitatif. Les contraintes empiriques de l'interprétation socio-anthropologique, Louvain-la-neuve, Academia Bruylant, 2008, p. 11 
enquêtes font état des statuts socio-économiques (riches, moyens et pauvres). Parmi les 24 ménages riches de l'étude de 2008, j'en ai retrouvé 13. Ceux-ci ont accepté de se soumettre à nouveau aux enquêtes. Pour les catégories "moyen » et "inférieur ", j'ai dû sélectionner des ménages manquants dans les mêmes espaces urbains en vue de disposer de la même configuration que celle de l'étude de 2008.

Pourquoi n'ai-je pas tiré tous les ménages au hasard ? Sans préjugés, certains indicateurs objectifs déterminent le statut économique d'un ménage, notamment la taille du ménage, le type de logement, etc. ${ }^{16}$ En tirant les ménages au hasard, je courais le risque de ne pas avoir les différentes catégories de la population étant donné que mon échantillon n'est pas très grand. Je devais veiller sur les 57 restants pour ne pas surestimer les ménages riches dans mon échantillon.

À l'instar de l'enquête précédente, j'ai recouru aux mêmes critères de calcul des indices de richesse pour une meilleure catégorisation. En ce qui concerne la catégorisation des ménages selon les statuts socio-économiques, j'ai établi la grille d'évaluation suivante :

- 2 points : être en possession d'une automobile ou d'une maison construite " en dur " au niveau des murs et du sol;

- 1 point : une moto, un salon (assortiment de fauteuils), un buffet/vitrine (meuble d'exposition dans le salon), une salle à manger (table et chaises destinées au repas), un frigo, une $\mathrm{TV}$, une résidence secondaire;

- 0,5 point : un vélo, une radio, la propriété d'une maison en matériaux bon marché (adobe, briques sans ciment, sol en terre battue...);

- 0,25 point : GSM et chaque lit que possède le ménage.

Cet indice de richesse a été calculé pour les 70 ménages, faisant apparaître des valeurs comprises entre 0,3 (un seul lit) et 13,5 (un foyer possédant tous les biens, dont 6 lits, à l'exception de la moto et de la seconde propriété).

16 Cf. Pierre Petit, op. cit. 


\subsection{Posture de recherche dans la collecte des données}

La démarche ethnographique est un parcours jalonné d'embûches et d'impasses. Elle nécessite une permanente vigilance et un savoir-faire de l'enquêteur pour pénétrer l'intimité des enquêtés. Certains auteurs parlent d'une sensibilité pragmatique en considérant qu'une des qualités essentielles du chercheur qualitatif réside dans sa capacité à réagir en adéquation avec le terrain, la sensibilité est alors associée à une forme de sagesse ${ }^{17}$. Pour d'autres, la posture sensible réside dans la capacité du chercheur à se saisir des subtilités des données issues du terrain ${ }^{18}$.

Ce sont notamment des rapports de genre, la précarité de certains informateurs, les faux rendez-vous, etc. De ces difficultés, découlent certaines prédispositions sociales (hospitalité, les enquêtés sont habitués aux entretiens) que je mobilisais pour m’intégrer dans les ménages. Aussi, je me saisissais de certaines opportunités afin d'accéder aux informations considérées parfois comme impénétrables.

C'est ainsi qu'il m'arrivait de partager une bière avec mes enquêtés. Dans cette proximité, certains informateurs me relataient leur vie conjugale. De même, mon statut matrimonial (marié) facilitait l'entretien sur certains domaines de la vie domestique. Ce sont notamment les questions liées à l'échange. Je recourais à ces diverses astuces pour vaincre la loi du silence.

Aussi, le caractère intensif de mes enquêtes m'offrait la possibilité d'assister aux aides cachées. Tel est le cas d'Annie (M.28). Je m'entretenais avec elle, subitement, l'une de ses sœurs est arrivée pour un "dépannage ». Elle lui avait donné de l'argent, de la farine de maïs et du poisson frais.

17 Catherine Cassel et al., "Learning to Be a Qualitative Management Researcher ", Management Learning, vol. 40, n 5, 2009, p. 513-532.

18 Barney G. Glaser, Theoretical Sensitivity, Mill Valley (CA), Sociology Press, 1978; Anselm Strauss et Juliet Corbin, Basics of Qualitative Research: Grounded Theory Procedures and Techniques, Newbury Park (CA), Sage Publications, 1990. 


\section{Caractéristiques socioéconomiques}

Je ne rapporte pas dans cette rubrique tous les éléments d'une analyse sociodémographique (volume de la population échantillonnée, la composition des ménages, etc.). Je me contente de quelques données susceptibles de favoriser mon interprétation des faits.

\subsection{Statut matrimonial}

La grande majorité de mes informateurs sont mariés (voir le tableau 1). Six enquêtées sont veuves. Elles ont une expérience de vie conjugale. Ces dernières, partant de cette expérience, m'ont permis de saisir certaines réalités sociales surtout lors du décès de leur mari. Chez les hommes, un informateur est polygame. Les coépouses ne vivent pas dans la même parcelle. Le faible taux de la polygamie ne signifie nullement pas que le phénomène n'existe pas ou tend à disparaître au profit de la monogamie. La polygamie existe sous sa version urbaine communément appelée « bureau $^{19}$ ».

\section{Tableau 1}

Répartition des informateurs par sexe, statuts matrimonial et socioéconomique

\begin{tabular}{|c|c|c|c|c|c|c|c|}
\hline \multirow{2}{*}{ Type d'assistance } & \multirow{2}{*}{$\begin{array}{c}\text { Échantillon } \\
\text { global } \\
(n=70)\end{array}$} & \multicolumn{2}{|c|}{$\begin{array}{l}\text { Niveau de vie } \\
\text { sup. }(n=13)\end{array}$} & \multicolumn{2}{|c|}{$\begin{array}{l}\text { Niveau de vie } \\
\text { moyen }(n=36)\end{array}$} & \multicolumn{2}{|c|}{$\begin{array}{c}\text { Niveau de vie } \\
\text { inf. }(n=21)\end{array}$} \\
\hline & & $\mathbf{F}$ & M & $\mathbf{F}$ & M & $F$ & M \\
\hline Marié-e-s monogames & $63(90,0 \%)$ & 7 & 3 & 19 & 14 & 11 & 9 \\
\hline Polygames & $1(1,4 \%)$ & - & 1 & - & - & - & - \\
\hline Veuves & $6(8,6 \%)$ & 2 & - & 3 & - & 1 & - \\
\hline
\end{tabular}

19 Aderanti Adepoju et Wariara Mbugua, «Les Mutations de la famille africaine ", dans Aderanti Adepoju (dir.), La Famille africaine. Politiques démographiques et développement, Paris, Karthala, coll. "Questions d'enfances", 1999, p. 59-84; Daniel Vangroenweghe, Sida et sexualité en Afrique, Anvers, Éditions EPO, 2000. 


\subsection{Structure par âge et par sexe}

Au regard du tableau 2, il s'agit d'une réalité des âges typique des pays en voie de développement. Mes informateurs sont jeunes. L'âge des informateurs serait un facteur déterminant dans l'exercice des dons cachés. Les jeunes conjoints auraient tendance à vouloir s'affirmer auprès de leurs proches parents. Ils chercheraient à prouver qu'ils sont responsables. Plus l'âge avance, plus la priorité serait accordée au ménage. Les conjoints avancés en âge cherchent à être assistés par leurs enfants qu'ils ne pensent à aider dans le caché.

Tableau 2

Répartition des informateurs par sexe, par statut socioéconomique et par tranches d'âge

\begin{tabular}{|c|c|c|c|c|c|c|c|}
\hline \multirow[t]{2}{*}{$\begin{array}{l}\text { Tranches } \\
\text { d'âge }\end{array}$} & \multirow{2}{*}{$\begin{array}{l}\text { Échantillon } \\
\text { global } \\
(\mathrm{n}=70)\end{array}$} & \multicolumn{2}{|c|}{$\begin{array}{l}\text { Niveau de vie } \\
\text { sup. }(n=13)\end{array}$} & \multicolumn{2}{|c|}{$\begin{array}{c}\text { Niveau de vie } \\
\text { moyen }(n=36)\end{array}$} & \multicolumn{2}{|c|}{$\begin{array}{c}\text { Niveau de vie } \\
\text { inf. }(n=21)\end{array}$} \\
\hline & & $\mathbf{F}$ & $\mathbf{M}$ & $F$ & M & $\mathbf{F}$ & M \\
\hline $20-24$ & $4(5,7 \%)$ & 1 & - & 1 & - & 2 & \\
\hline $25-29$ & $9(12,9 \%)$ & 2 & - & 2 & - & 3 & 2 \\
\hline $30-34$ & $12(17,1 \%)$ & 2 & - & 4 & 1 & 2 & 3 \\
\hline $35-39$ & $14(20,0 \%)$ & 1 & 1 & 5 & 3 & 2 & 2 \\
\hline $40-44$ & $11(15,7 \%)$ & 1 & 1 & 3 & 4 & 1 & 1 \\
\hline $45-49$ & $8(11,4 \%)$ & 1 & - & 3 & 2 & 1 & 1 \\
\hline $50-54$ & $6(8,6 \%)$ & 1 & - & 2 & 2 & 1 & - \\
\hline $55-59$ & $3(4,3 \%)$ & - & 1 & 1 & 1 & - & - \\
\hline $60-64$ & $3(4,3 \%)$ & - & 1 & 1 & 1 & - & - \\
\hline Total & $70(100,0 \%)$ & 9 & 4 & 22 & 14 & 12 & 9 \\
\hline
\end{tabular}

\subsection{Activités professionnelles}

Contrairement aux décennies passées où les ménages lushois survivaient grâce au salaire. Actuellement, ces ménages rentrent dans ce que Georges Mulumbwa Mulumbwa et Jerry Kalonji wa Mpoyo qualifient de génie créateur du Lushois ${ }^{20}$, c'est-à-dire de

$\overline{20}$ Georges Mulumbwa Mutambwa et Jerry Kalonji wa Mpoyo, «L'économie morale : Approche lexicale ", dans Pierre Petit (dir.), Lubumbashi 2000. La situation des ménages dans une économie de précarité, Lubumbashi, Observatoire du 
l'effritement de l'emploi salarié qui « a fini par mettre sur pied quantité de stratégies de survie qui s'étalent sur un continuum allant d'un job légitime aux activités aussi criminelles que juteuses en passant par tout un réseau de pratiques prohibées qui consacrent ainsi l'émergence de l'informel dans une ville minière dont la population basa longtemps son existence sur le salariat ".

Dans le tableau 3, il s'observe une importante part des activités commerciales. Celles-ci, par leurs rentrées journalières, permettent aux ménages d'assurer la survie des membres. Ces commerces sont majoritairement informels, c'est-à-dire liés à la crise, dont ils sont la dramatisation et le reflet. Ils sont suivis de travaux de femmes qui n'ont pas d'activités lucratives. Ces femmes dépendent totalement des rentrées financières de leur mari. Viennent ensuite, les informateurs qui s'adonnent aux petits métiers. En ne prenant que ces catégories, il y a lieu de se faire une idée sur les échanges cachés. Etant donné que chaque conjoint voudrait assister ses proches, chacun essaiera de ponctionner dans ce qu'il gagne ou reçoit pour répondre à ses obligations d'assistance. 


\section{Tableau 3}

Répartition des informateurs par sexe, par statut socioéconomique et par activités lucratives

\begin{tabular}{|c|c|c|c|c|c|c|c|}
\hline \multirow{2}{*}{ Statut socioéconomique } & \multirow{2}{*}{$\begin{array}{c}\text { Échantillon } \\
\text { global } \\
(n=70)\end{array}$} & \multicolumn{2}{|c|}{$\begin{array}{l}\text { Niveau de vie } \\
\text { sup. }(n=13)\end{array}$} & \multicolumn{2}{|c|}{$\begin{array}{l}\text { Niveau de vie } \\
\text { moyen }(n=36)\end{array}$} & \multicolumn{2}{|c|}{$\begin{array}{c}\text { Niveau de vie } \\
\text { inf. }(n=21)\end{array}$} \\
\hline & & $\mathrm{F}$ & M & $\mathbf{F}$ & M & $\mathrm{F}$ & M \\
\hline Inactifs/ménagères & $15(21,4 \%)$ & 4 & - & 5 & - & 6 & - \\
\hline $\begin{array}{l}\text { Petits métiers, ouvriers } \\
\text { indépendants }\end{array}$ & $13(18,6 \%)$ & - & - & 2 & 4 & 1 & 6 \\
\hline $\begin{array}{l}\text { Ouvriers non spécialisés } \\
\text { salariés }\end{array}$ & $\mathbf{5}(7,1 \%)$ & - & - & 2 & 3 & & - \\
\hline $\begin{array}{l}\text { Commerce et activités } \\
\text { commerciales }\end{array}$ & $25(35,7 \%)$ & 3 & 1 & 11 & 3 & 5 & 2 \\
\hline Ouvriers qualifiés salariés & $2(2,9 \%)$ & - & 1 & & 1 & - & - \\
\hline Employés, fonctionnaires & $7(10,0 \%)$ & 1 & - & 2 & 3 & - & 1 \\
\hline Cadres, professions libérales & $3(4,3 \%)$ & 1 & 2 & - & - & - & - \\
\hline Total & $70(100,0 \%)$ & 9 & 4 & 22 & 14 & 12 & 9 \\
\hline
\end{tabular}

Les quelques réalités socioéconomiques montrent que les informateurs sont jeunes et surtout monogames. Ils sont engagés dans les activités lucratives informelles plus que formelles. En tant que jeunes, d'une part, ils ont l'obligation morale de rendre aux aînés les bienfaits reçus, et, d'autre part, ils veulent s'affirmer auprès de leurs parents, prouver qu'ils sont responsables et capables de supporter les autres.

À ceci s'ajoute le poids des rapports de genre. Le mari, comme chef de ménage, cherche toujours à extérioriser qu'il est au-dessus de l'épouse. Pour ce faire, il assiste ses parents en coulisses. L'épouse, de son côté, valorise son mariage. Cela passe par l'assistance régulière des proches parents vivant en dehors de son ménage. Tout ceci m’amène à parler des dons cachés. 


\section{Situations d'échange des dons cachés}

Les aides cachées ne sont pas exclusives. Certains conjoints combinent les dons formels et informels. D'autres n'en font que d'un type. Les échanges impliquent les conjoints de différents statuts socioéconomiques. Le tableau 4 en donne les détails.

Tableau 4

Répartition des informateurs par sexe, par statut socioéconomique et par types de dons

\begin{tabular}{|c|c|c|c|c|c|c|c|}
\hline \multirow{2}{*}{ Type d'assistance } & \multirow{2}{*}{$\begin{array}{l}\text { Échantillon } \\
\qquad \begin{array}{l}\text { global } \\
(n=70)\end{array}\end{array}$} & \multicolumn{2}{|c|}{$\begin{array}{l}\text { Niveau de vie } \\
\text { sup. }(n=13)\end{array}$} & \multicolumn{2}{|c|}{$\begin{array}{l}\text { Niveau de vie } \\
\text { moyen }(n=36)\end{array}$} & \multicolumn{2}{|c|}{$\begin{array}{c}\text { Niveau de vie } \\
\text { inf. }(n=21)\end{array}$} \\
\hline & & Épouse & Mari & Épouse & Mari & Épouse & Mari \\
\hline Transfert des dons officiels & $25(35,7 \%)$ & $3(7,0 \%)$ & $2(7,4 \%)$ & $6(14,0 \%)$ & $4(14,8 \%)$ & $2(4,7 \%)$ & $8(29,6 \%)$ \\
\hline Transfert des dons cachés & $14(20,0 \%)$ & $2(4,7 \%)$ & $1(3,7 \%)$ & $5(11,6 \%)$ & $3(11,1 \%)$ & $2(4,7 \%)$ & $1(3,7 \%)$ \\
\hline $\begin{array}{l}\text { Transfert des dons officiels et } \\
\text { cachés }\end{array}$ & $31(44,3 \%)$ & $4(9,3 \%)$ & $1(3,7 \%)$ & $11(25,6 \%)$ & $7(25,9 \%)$ & $8(18,6 \%)$ & - \\
\hline
\end{tabular}

En prenant les informateurs dans leur catégorie, il transparait que l'écart n'est pas grand. Le mari et l'épouse s'adonnent aux échanges cachés presque dans la même proportion en fonction de leur statut socioéconomique. Il s'observe néanmoins, dans la catégorie des conjoints alliant les dons officiels et cachés, une relative prédominance des épouses de ménages riches et pauvres. Pourquoi les conjoints se livrent-ils aux dons cachés ?

\subsection{Motivations des dons cachés}

Les motivations des aides cachées sont diverses. Elles sont déterminées par les normes matrimoniales et les règles d'héritage. Ces préceptes varient avec le statut socioéconomique du ménage et d'autres déterminants sociaux comme la réciprocité, la valorisation de soi, le manque de confiance entre les conjoints, etc.

\subsubsection{Réciprocité et valorisation sociale}

Servons-nous du témoignage de Séraphine (M.70) pour comprendre la représentation de soi que recherchent les conjoints auprès de leur famille. 
Ma belle-mère et mes beaux-frères survivent grâce à l'assistance mensuelle de mon mari. Comme mes parents sont salariés, mon mari ne les assiste presque pas. Il faut une urgence pour qu'il intervienne financièrement. Rarement, l'initiative est venue de lui pour aider mes parents. Cela me déplaît car c'est une injustice dans la distribution des dons. Par les gestes qu'il pose envers sa famille, il est respecté et apprécié. J'ai également besoin qu'on m'apprécie par mes proches parents. Pour ce faire, j'assiste secrètement mes parents. De l'argent que me donne mon mari pour la ration alimentaire, j'en défalque une partie que j'envoie à ma famille. Je m'arrange avec ce qui reste pour subvenir aux besoins de mon ménage. Aussi, mon mari ne me parlait plus de ses rentrées financières. Il me donnait de l'argent pour le budget sans autres explications. Un jour, il avait oublié mille dollars américains dans l'une des poches de son costume. J'en avais soutiré trois cents que j'avais envoyés à ma mère.

Ce témoignage montre que la réciprocité ne tient pas absolument au contre-don. Elle est déterminée également par la représentation symbolique. Chaque conjoint, au-delà de rendre les bienfaits reçus des aînés, tient au prestige social. Cela passe par les aides accordées aux proches parents. Ces assistances envers les familles d'origine ne tiennent pas compte du statut socioéconomique des bénéficiaires. Si la famille de l'un des époux est démunie, ce n'est pas une raison pour qu'elle bénéficie davantage des dons que celle qui est bien pourvue. La réciprocité revêt une double obligation morale : la logique de la dette et la valorisation sociale. La dette dans ce sens que chaque conjoint « reste toujours l'enfant de ses parents. Et malgré tout, un enfant qui finit, au moins formellement, par être reconnaissant ${ }^{21}$ ». Et Monique Pontault de renchérir qu'en « recevant la vie, l'individu contracte, par ce fait même, une dette envers ses géniteurs ${ }^{22}$ ». Dans cette logique, les conjoints rendent les bienfaits reçus à travers divers gestes de générosité (la prise en charge des cadets, les dons en

21 Anne Muxel, Individu et mémoire familiale, $2^{\mathrm{e}}$ édition, Paris, Nathan, 2002, p. 36.

22 Monique Pontault, Frères de sang, saurs de lait. Anthropologie d'une marginalisation familiale et sociale, Paris, L'Harmattan, 2001, p. 215. 
nature et en espèces, etc.). Il y a aussi le prestige social que recherchent les conjoints auprès de leurs parents. Ayant quitté les parents pour fonder leur ménage, les conjoints cherchent à prouver qu'ils sont devenus responsables. La meilleure manière de l'affirmer est de s'occuper des intérêts des autres par des dons cachés.

\subsubsection{Manque de confiance et soupçons mutuels des conjoints}

Des discours des informateurs, je relève une accusation mutuelle entre les conjoints. Les maris reprochent aux épouses d'être trop attachées à leur famille d'origine. Elles assistent leurs parents à l'insu des maris (M.55). Les épouses, quant à elles, dénoncent les actions illicites des maris. Elles estiment que l'argent que gagnent les maris n'est pas rapporté totalement au ménage (M.21). Ils en soutirent le tiers (kuricomeka ${ }^{23} /$ litt. se brancher ou kukata kipande/amputer d'une partie), et les deux tiers sont remis à l'épouse. D'autres maris ne disent pas à leur épouse le montant exact de leur salaire (M.14). L'argent qu'ils lui remettent est considéré comme étant le salaire mensuel.

Dans cet univers des soupçons, chaque conjoint croit ne pas faire davantage pour ses parents. De ce fait, il s'adonne aux dons cachés afin de privilégier ses parents. Le cas de Karel (M.33) est édifiant à ce propos.

Certains hommes ne pensent qu'à plaire leur belle-famille. Ils oublient leurs proches parents. Je prends mon cas, j'avais versé des dollars américains et des biens matériels en guise des compensations matrimoniales. J'héberge la petite sœur de mon épouse. Celle-ci envoie régulièrement de l'argent et des biens dans sa famille résidant à Kigoma. Dans tout ça, qui gagne ? Je

23 Les deux concepts traduisent le fait de soutirer une partie d'argent que l'on cache judicieusement pour que le partenaire ne le découvre pas. Kucomeka est couramment utilisé dans les transports en commun. Le convoyeur (la personne qui fait payer les passagers dans le bus) est payé en fin de journée par le chauffeur. Le plus souvent, il perçoit les frais de la dernière course (soit 5000 francs congolais). Pour maximiser, à chaque trajet, il soutire au moins $250 \mathrm{FC}$, qu'il introduit dans un sous-vêtement. Cette action n'est autre que kucomeka. 
ne suis pas distrait. Dans ce que je gagne comme argent, j'en garde une partie afin d'assister secrètement mes parents.

Le mari aide ses proches parents avec l'argent des rentrées de ses extra-muros ${ }^{24}$. L'argent relevant des activités officielles est remis à l'épouse. Cette attitude du mari prouve à l'épouse que tout ce qu'il gagne comme argent est injecté dans l'économie du ménage. Comme le revenu des activités informelles n'est pas connu de l'épouse, le mari a une marge de manœuvre pour subvenir secrètement aux besoins de ses apparentés. L'épouse, quant à elle, réaménage les dépenses de son budget avec l'argent qu'elle perçoit du mari. Elle agit de manière à ce que le mari ne constate pas des retenues faites.

Ce manque de confiance me conduit à Erving Goffman dans sa représentation dramaturgique. Cet auteur distingue deux régions dans lesquelles évoluent les comportements humains, à savoir : antérieure et postérieure. Il constate que, dans " une représentation, on accentue l'expression de certains aspects tandis que l'on en dissimule d'autres qui pourraient discréditer l'impression produite. Il est évident que les faits accentués font leur apparition dans ce que l'on appelle la région antérieure... une région postérieure ou coulisse où l'on peut observer les faits dissimulés ${ }^{25}$ ". Comme on le remarque, la région antérieure est celle de la performance et de la valorisation de soi alors que, dans la région postérieure, à laquelle le public n'a pas accès, l'acteur a toute latitude de contredire l'impression produite par la représentation sur la scène.

Les aides cachées ne s'écartent pas de cette logique. Sur la sphère publique, les conjoints affichent leur solidarité envers les deux familles. Ils accueillent d'autres parents, ils distribuent officiellement des dons aux différents membres de la famille

24 Dans une économie où le revenu mensuel des activités du secteur formel ne permet pas de terminer le mois, la plupart des Lushois, pour combler le manque, recourent à une série d'activités en dehors du secteur formel, qui rapportent des dividendes pour le ménage. Ces rentrées d'activités extraformelles ne sont pas contrôlées par les épouses. En pratique, l'extra-muros rapporte davantage que le revenu mensuel.

25 Erving Goffman, La Mise en scène de la vie quotidienne, Paris, Éditions de Minuit, 1973, p. 110. 
vivant en dehors de leur ménage, etc. Sur le plan postérieur, les logiques d'échange sont en contradiction avec les solidarités formelles. C'est dans cette zone que les conjoints se soupçonnent mutuellement. Chacun cherche à accorder beaucoup d'avantages à ses proches parents. Qu' est-ce qui justifieraient ces comportements de conjoints? J'estime que les dons cachés relèvent aussi des rapports prescriptifs de genre.

\section{Logiques matrimoniales dans l'exercice des dons cachés}

Les rapports sociaux sont dominés par une emprise masculine à Lubumbashi. Le mari reste le chef de ménage. Il lui revient d'entretenir son épouse, d'assumer toutes les charges du ménage et d'entretenir sa belle-famille. Un mari qui ne s'occupe que de sa famille d'origine et de son foyer n'est pas considéré par sa belle-famille.

\subsection{Subordination de la femme à l'homme}

Revenons au témoignage de Séraphine. Son mari transgresse les règles voulues dans les interactions entre le gendre et sa bellefamille. Il aide fréquemment ses proches parents que ceux de sa belle-famille. Si Séraphine ne recourait pas aux aides cachées, elle serait prise pour une mauvaise fille par ses parents. Son mariage serait qualifié du nom "bukweri bwa njina " parce qu'il n'y a pas de retombées dans sa famille d'origine. On attend de l'épouse une particulière attention à sa famille d'origine. Quand une épouse n'assiste pas ses parents ou qu' elle investit tout son argent dans son ménage, elle est critiquée en ces termes : weye uko wa nini? (litt. toi, tu es de quoi?). Pour dire qu'elle ne vaut pas grand-chose parce qu'elle ne s'occupe que de son ménage sans aucune réciprocité pour sa famille d'origine. En d'autres termes, elle est pumbafu (imbécile).

Ces critiques ne sont pas formulées contre le mari. La société tolère que le mari se préoccupe prioritairement de sa famille nucléaire. D'où l'expression swahili kuzala mwanaume ni kupotesha (accoucher d'un garçon, c'est perdre). On entend par là que les hommes, dès qu'ils se marient, ne s'intéressent presque plus 
à leur famille d'origine. Ils s'occupent davantage de leur foyer et de leur belle-famille. Ce qui n'est pas le cas pour l'épouse. Celle-ci y est attachée. Michel (M.9) justifie cette attitude de la femme en s'inspirant d'un passage biblique.

La bible est claire dans les rapports entre les conjoints avec leurs familles d'origine. Dieu dit : l'homme quittera ses parents et $s^{\prime}$ attachera à sa femme ${ }^{26}$. On ne dit pas que la femme quittera sa famille et ses parents. Il est donc normal que les femmes soient plus attachées à leur famille que les hommes. Et quand je m'attache à ma femme, cela signifie autrement que je me lie à sa famille parce qu'elle ne l'a pas quittée.

Le discours de cet informateur est intéressant. Il expose les logiques matrimoniales d'échange entre les conjoints. Pour lui, l'épouse ne quitte ses parents que physiquement. Son esprit y est attaché. De ce fait, elle n'a pas à investir dans le ménage qui ne lui appartient pas. Par contre, l'homme qui s'unit à elle doit veiller au bien-être de la famille qui l'accueille. Dans cette logique, il ne revient pas à l'épouse de supporter les charges familiales. Son apport surtout financier ne peut être que secondaire "kusaidiya» (M.68). Si l'épouse est salariée, le mari n'a pas à s'ingérer dans ses dépenses. S'y intéresser, c'est perdre de son autorité et inverser le rôle social au sein du ménage. Ce qui appartient à l'épouse, c'est d'abord pour elle, sa famille d'origine et enfin son foyer. Les normes coutumières sont claires à ce niveau. Elles indiquent que la femme est sous l'autorité du mari, mais qu' elle ne lui appartient pas.

L'attachement de l'épouse à sa famille est donc normal, car l'« époux n'avait obtenu, en échange des biens matrimoniaux, qu'un droit sur la progéniture et sur les activités domestiques de sa femme, certainement pas sur sa personne dans son ensemble, et la femme reste incontestablement membre à part entière de son lignage et de sa famille jusqu'à son $\operatorname{trépas}^{27}$ ".

$\overline{26}$ Cf. les versets bibliques (Genèse, 2 :24; Matthieu, 19 :5; Marc 10 :7).

27 Joël Noret et Pierre Petit, Mort et dynamiques sociales au Katanga (République Démocratique du Congo), Paris, L'Harmattan, 2011, p. 90. 
Les épouses éveillées profitent du vivant de leur mari pour faire bénéficier leurs parents des avantages de leur ménage. Elles combinent ainsi les solidarités formelles et informelles. Dans le cas des solidarités formelles, le don est accompagné d'une valorisation de soi. L'un des conjoints (ou les deux) apporte directement l'aide aux beaux-parents et s'exprime en ces termes "leo turimikumbuka ako" (aujourd'hui, je me suis souvenu de vous). Ce geste de bienfaisance prend souvent la forme d'une manifestation de respect ou d'affection, en même temps qu'il est motivé par le désir d'indiquer à la belle-famille que c'est une bru ou un gendre attentionné. Si les conjoints ne peuvent pas se déplacer pour apporter leur don, il leur est possible de le confier soit au parent hébergé, soit à l'un des enfants du ménage qui le porterait aux bénéficiaires. Cette assistance est inclusive car les conjoints consentent à la matérialisation de l'aide. Dans le cas des solidarités informelles, il s'agit d'actions relevant de l'assistance cachée. Le bienfaiteur s'arrange pour qu'il n'y ait aucun témoin lorsque le don est effectué. La stratégie a pour finalité de se prémunir contre toute dénonciation. En donnant l'aide, le conjoint précise au bénéficiaire que l'assistance relève de son initiative. Elle ne doit pas être rapportée au partenaire abusé. "Iyi ni yako, ushimwambiye mutu "(ça c'est pour toi, ne dis à personne) est l'expression couramment utilisée pour prévenir le bénéficiaire. Ces précautions sont prises pour que le partenaire abusé ne le sache pas. Dans ce cas, le donateur et le bénéficiaire deviennent complices. Lorsque le bénéficiaire déroge du principe, il expose son bienfaiteur aux conflits et réduit sa possibilité d'être assisté à nouveau.

Le caractère illicite de cet échange est qualifié de John Cena par certains informateurs. John Cena est un catcheur américain. Cette star, quand elle terrasse son adversaire, avant de le soumettre à sa prise finale, lui passe la paume de sa main sur le visage. Ce rituel est connu sous le slogan you can't see me (tu ne peux pas me voir). Cette réalité du sport a été introduite dans les réalités domestiques pour désigner les dons cachés. 
Cette analogie à John Cena par certains enquêtés me pousse à considérer les conjoints comme étant des adversaires plutôt que des partenaires. Â la différence du catch, les conjoints ne se placent pas dans une position de combat. Seulement, chacun ignore les actions que pose son partenaire dans le caché. Le conjoint abusé est comme ce catcheur inconscient. Il ne voit pas l'échange qui s'effectue alors qu'il est sur la scène.

L'efficacité des dons cachés réside dans la sélection du bénéficiaire. Il s'agit le plus souvent des géniteurs. Les beaux-parents et le partenaire abusé ont une relation d'évitement caractérisée par le respect. Partant de ce type de rapports sociaux, la victime n'a pas la possibilité de soutirer les informations sur les aides cachées.

Dans mes enquêtes, les biens matériels et l'argent circulent davantage entre les membres apparentés. Le tableau 5 en donne les détails.

\section{Tableau 5}

Répartition des informateurs par sexe, par statut socioéconomique et par nature d'aides

\begin{tabular}{|c|c|c|c|c|c|c|c|}
\hline \multirow{2}{*}{ Type d'assistance } & \multirow{2}{*}{$\begin{array}{c}\text { Échantillon } \\
\text { global } \\
(n=70)\end{array}$} & \multicolumn{2}{|c|}{$\begin{array}{l}\text { Niveau de vie } \\
\text { sup. }(n=13)\end{array}$} & \multicolumn{2}{|c|}{$\begin{array}{l}\text { Niveau de vie } \\
\text { moyen }(n=36)\end{array}$} & \multicolumn{2}{|c|}{$\begin{array}{l}\text { Niveau de vie } \\
\text { inf. }(n=21)\end{array}$} \\
\hline & & Épouse & Mari & Épouse & Mari & Épouse & Mari \\
\hline Argent & $29(41,4 \%)$ & $3(7,0 \%)$ & $3(11,1 \%)$ & $8(18,6 \%)$ & $6(22,2 \%)$ & $2(4,7 \%)$ & $7(25,9 \%)$ \\
\hline Aliments & $10(14,3 \%)$ & $1(2,3 \%)$ & - & $3(7,0 \%)$ & $1(3,7 \%)$ & $5(11,6 \%)$ & - \\
\hline Argent et aliments & $13(18,6 \%)$ & $1(2,3 \%)$ & - & $4(9,3 \%)$ & $3(11,1 \%)$ & $5(11,6 \%)$ & - \\
\hline Argent et biens matériels & $18(25,7 \%)$ & $4(9,3 \%)$ & $1(3,7 \%)$ & $7(16,3 \%)$ & $4(14,8 \%)$ & - & $2(7,4 \%)$ \\
\hline
\end{tabular}

Ce tableau reprend les différents transferts. Il ne concerne pas exclusivement les dons cachés. Comme on peut le remarquer, le mari donne davantage de l'argent. L'épouse allie l'argent et les aliments. De même, elle est plus engagée dans le transfert des biens matériels. Dans une économie de précarité, ce ne sont pas tous les jours que les apparentés ont à manger. Pendant la crise, ils sollicitent une assistance à leur parente qui peut leur donner 
une part de ses provisions. Elle ne le dira pas au mari. Il en ressort que chaque partenaire profite de sa position pour aider ses proches.

\subsection{Dons cachés comme économie du symbolisme}

Dans cette conception, lorsqu' un conjoint vole son partenaire ou aide secrètement ses parents, il agit prioritairement dans le sens d'asseoir sa valeur sociale auprès de ses parents. Pour certains informateurs (M.45, 69), il ne s'agit pas d'un cynisme économique. On est dans une économie du symbolisme et de la représentation de soi. Ainsi, Séraphine, en volant l'argent de son mari, cherche à préserver la bonne image chez ses parents qu'elle est une bonne fille. Elle est attentive et n'oublie pas sa famille d'origine.

Il en est de même du mari qui ne rapporte pas tout argent qu'il gagne parce qu'il doit résoudre discrètement certains problèmes liés à sa famille d'origine. Il ne doit pas toujours apporter cette aide de manière officielle. Cela est considéré par ses parents comme une incapacité de ne pas gérer les affaires familiales sans associer l'épouse. Il sera alors qualifié de «dominé par l'épouse ». Pour extérioriser leur indépendance, certains hommes agissent dans les coulisses.

Les aides cachées sont réfléchies. Les ponctions faites ne bouleversent pas l'équilibre du ménage de manière remarquée. J'y dégage une certaine morale quant aux limites dans les actions posées par les conjoints. Ces époux n'agissent absolument pas au détriment de leur ménage, bien que leurs actions aient des conséquences négatives sur leur budget. Ils ont des seuils et se soucient des mauvaises retombées qui adviendraient de leur ponction. La précaution prise dans le prélèvement montre qu'il y a là une forme d'appréhension morale dans leurs actions.

Dans l'échange caché, l'aide est unilinéaire, c'est-à-dire qu'elle va des enfants vers leurs géniteurs. Ces assistances sont dirigées presque exclusivement vers les géniteurs et les parents collatéraux. La fréquence des prestations cachées n'est pas maîtrisée. Elle dépend des possibilités financières et matérielles des conjoints. 
Comme on le remarque, il n'y a pas que les logiques matrimoniales qui détermineraient les aides cachées. Je relève aussi la problématique d'accès à l'héritage. La société ne reconnaît presque pas l'apport de l'épouse dans les richesses accumulées par les conjoints. En cas de décès, l'épouse est souvent préjudiciée par les membres de la belle-famille. Pour se prémunir, elle investit dans sa famille d'origine.

\section{Règles d'héritage : un des déterminants des échanges cachés}

Dans la représentation collective, l'épouse est constamment perçue par sa belle-famille comme une étrangère. Cela est perceptible lors de décès du mari. Elle est souvent maltraitée et accusée d'être responsable de la mort du mari ${ }^{28}$. Après l'enterrement du mari, il arrive que la veuve soit renvoyée sans héritage. Les membres de la belle-famille acceptent à la limite qu' elle emporte les ustensiles de cuisine. Jadis, pour protéger la progéniture du défunt et ses biens, la coutume encourageait le lévirat.

6.1. Épouse perçue comme une étrangère

À Lubumbashi, cette pratique coutumière n'est pas une norme prescriptive. La dignité de la femme est valorisée. Plusieurs organisations sociales sensibilisent des veuves à ne pas se soumettre au lévirat; ce sont des Églises et des organisations non gouvernementales. Dans ce cas, la belle-famille ne garde pas la veuve après le conseil de famille. Elle est renvoyée dans sa famille d'origine afin qu'elle y refasse sa vie.

Dans beaucoup de cas, le deuil d'un mari est précédé d'un conflit. Certains informateurs (M.32, 61) considèrent ce comportement de la belle-famille comme une stratégie pour dépouiller la veuve des biens laissés par son défunt mari. Pour ce faire, elle crée un conflit afin que la négociation soit difficile. Quelques veuves déplorent l'ingratitude des membres de la belle-famille.

$\overline{28}$ Sylvie Ayimpam, "Violences sociales et exclusions réciproques en milieu africain. Le cycle des échanges de violence dans les rapports familiaux ", dans Jacky Bouju et Mirjam de Bruijn (dir.), Violences sociales et exclusions. Le développement social de l'Afrique en question, Bulletin de l'APAD, $\mathrm{n}^{\text {os }}$ 27-28, 2005, p. 57-74. 
Du vivant de leurs maris, elles hébergeaient différents parents et leur distribuaient divers biens. Elles sont étonnées de constater que leurs bienfaits soient effacés du revers de la main. Le décès du mari provoque inéluctablement une crise entre les familles de conjoints. La parenté par alliance se désagrège. La séparation avec la veuve est parfois brutale. Certains membres de la belle-famille ne la fréquentent plus ou ne lui parlent plus.

L'agissement de certains membres de la belle-famille se justifie par les rapports de genre. Ces membres ne reconnaissent pas le travail de l'épouse dans la stabilité du ménage. Pour eux, tous les biens meubles et immeubles du ménage appartiennent au mari, même si l'épouse a contribué à leur acquisition.

Michel Bozon et François de Singly, parlant du travail de l'homme et de la femme, en distinguent deux types. Il y a, d'une part, le travail désintéressé, qui relève de la femme et, d'autre part, le travail de nécessité, celui de l'homme, à cause de son apport dans la stabilité du ménage. Ces auteurs s'attaquent à ces perceptions. Pour eux, il n'y a pas un travail plus important que l'autre $^{29}$. À Lubumbashi, dans les représentations sociales, le travail de la femme est dévalorisé.

\subsection{Dons cachés et sécurité sociale}

Face à ces réalités socioculturelles, l'épouse privilégie sa position de subordonnée dans le ménage. Si elle est salariée, son argent est investi dans le circuit social où elle estime avoir beaucoup de considération. C'est notamment dans sa famille d'origine. Quelles que soient les difficultés qu'elle éprouverait, ses proches parents ne l'abandonneront pas. Le mariage n'offre pas toute la sécurité sociale.

De ce fait, les épouses mobilisent les dons officiels et cachés parce qu'elles savent qu'en cas de décès, elles seront les perdantes. $\mathrm{Au}$ moins, ce qu'elles envoient comme biens matériels à leurs parents, aucun membre de la belle-famille n’irait les réclamer.

29 Michel Bozon et François de Singly, «Fortune et infortune de la femme mariée ", Population, 43e année, no 3, 1988, p. 683-685. 
Aussi, partant des règles matrimoniales et de mauvais traitements qui sont infligés aux femmes après le décès de leur mari, elles s'abstiennent d'investir dans les grands projets du ménage. Elles laissent cette responsabilité au mari, en tant que chef de ménage. Leur participation dans le ménage est secondaire. Le tableau 6 présente les appréciations de contributions dans le ménage selon les conjoints.

Tableau 6

Répartition des informateurs par sexe, par statut socioéconomique et par motivations d'aides

\begin{tabular}{|c|c|c|c|c|c|c|c|}
\hline \multirow{2}{*}{ Motivations } & \multirow{2}{*}{$\begin{array}{c}\text { Échantillon } \\
\text { global } \\
(n=70)\end{array}$} & \multicolumn{2}{|c|}{$\begin{array}{l}\text { Niveau de vie } \\
\text { sup. }(n=13)\end{array}$} & \multicolumn{2}{|c|}{$\begin{array}{l}\text { Niveau de vie } \\
\text { moyen }(n=36)\end{array}$} & \multicolumn{2}{|c|}{$\begin{array}{c}\text { Niveau de vie } \\
\text { inf. }(n=21)\end{array}$} \\
\hline & & Épouse & Mari & Épouse & Mari & Épouse & Mari \\
\hline $\begin{array}{l}\text { Investir à perte et injustice } \\
\text { sociale }\end{array}$ & $41(58,6 \%)$ & $8(18,6 \%)$ & & $21(48,8 \%)$ & $2(7,4 \%)$ & $10(23,3 \%)$ & - \\
\hline S'entraider entre les conjoints & $24(34,3 \%)$ & $1(2,3 \%)$ & $2(7,4 \%)$ & $1(16,3 \%)$ & $9(33,3 \%)$ & $2(4,7 \%)$ & $9(33,3 \%)$ \\
\hline Égoïsme féminin & $5(7,1 \%)$ & - & $2(7,4 \%)$ & & $3(11,1 \%)$ & - & - \\
\hline
\end{tabular}

Il se dégage que la majorité des informatrices $(90,7 \%$, soit 39/43) déplorent l'inégalité sociale dans le partage des biens pour lesquels les conjoints ont investi. Elles dénoncent l'usurpation des biens meubles et immeubles par les parents du mari lors de décès.

Le mari peut laisser un testament, il n'est jamais pris en compte lors du partage de l'héritage. À Lubumbashi, l'accès à l'héritage après le décès d'un mari s'inscrit toujours dans deux logiques et deux droits qui s'affrontent. "Traditionnellement en effet, ce sont les germains du défunt parfois aussi les oncles et tantes qui, en tant qu'aînés sociaux du lignage du mort, s'approprient la majeure partie de son patrimoine [...]. Mais face à cette norme favorable aux générations aînées s'érige aujourd'hui celle qui, héritée de la colonisation et relayée par le droit congolais, tend à reconnaître à la veuve et aux orphelins la position d'héritiers principaux $^{30}$.»

$\overline{30}$ Joël Noret et Pierre Petit, op. cit., p. 96. 
Malgré les campagnes de sensibilisation, l'héritage reste sous l'emprise de la coutume. Les tentatives de création de nouveaux espaces sociaux contribuent à accentuer les conflits entre les familles. Les droits et les intérêts des orphelins et de la veuve sont déterminés par les aînés du défunt même si un testament écrit explicite la clé de répartition. Lépouse et les orphelins peuvent bien organiser leur défense à l'héritage, ils finissent par être rattrapés soit par l'idiome de la sorcellerie soit par des conflits avec les aînés sociaux du défunt.

Les menaces de divers ordres finissent par les convaincre de renoncer à leur droit. Dans ce cas, ils attendent que les aînés fassent selon leur volonté. Pour s'en prémunir, les épouses se réservent d'investir de leur argent dans le ménage. Elles préfèrent être totalement prises en charge par le mari selon les normes prescriptives de genre. Entretemps, elles profitent de leur position privilégiée en tant que maîtresse du ménage pour aider leurs proches parents tant qu'elles le peuvent. Ainsi, les dons cachés ne visent qu'à renforcer leur sécurité auprès des parents.

\section{Conclusion}

Les aides cachées sont le revers des pratiques de la solidarité. Elles résultent de plusieurs facteurs socioculturels. Dans une économie de précarité, chaque conjoint est contraint de répondre aux obligations de solidarité, soit pour rendre les bienfaits reçus des aînés, soit pour l'honneur social. Au-delà du paradigme des solidarités communautaires, les dons cachés envers les familles de conjoints sont déterminés par les rapports prescriptifs de genre. Ces rapports sont sous-tendus par les normes matrimoniales et les règles d'héritage. Dans une société où les maris sont considérés comme étant les chefs de ménage, il leur revient d'assumer le rôle qui leur est dévolu. Ils doivent entretenir leur épouse et assister leur belle-famille.

Ces interventions leur valent une considération sociale et l'honneur auprès de leur belle-famille. Quand cette famille ne tire pas profit du mariage de leur parente, l'union relève de la honte. Les épouses, quant à elles, restent attachées à leur famille 
d'origine. Elles se considèrent comme des étrangères dans le ménage qu'elles fondent. Pour ce faire, elles ne sont pas motivées à y investir leur argent et les autres biens matériels. En cas de divorce ou de décès du mari, le partage des biens accumulés n'est jamais équitable. Pour se prémunir contre ces inégalités sociales, les épouses transferent divers dons dans leur famille d'origine. Elles agissent ainsi pour se protéger contre les incertitudes de l'avenir. Elles endetteraient ainsi leur famille d'origine, de sorte qu'en cas des problèmes dans leur ménage, les membres de leur famille ne puissent pas refuser de les soutenir et de les héberger. Il y a là une assurance symbolique que les épouses recherchent à travers les dons cachés.

Les droits et les intérêts des orphelins et de la veuve ne sont jamais respectés par les frères du défunt. Les normes coutumières dominent sur les droits écrits congolais. Le plus souvent, l'épouse s'en tient à la bonne volonté des aînés du défunt, même si le testament détermine la répartition de l'héritage. Quand l'épouse essaie de revendiquer ses droits, elle est rattrapée soit par l'idiome de la sorcellerie, soit par les conflits avec la belle-famille du défunt. Dans ce cas, l'épouse préfère garder sa position de subordonnée dans les interactions domestiques.

De même, le mari, qui est de pourvoyeur principal du ménage, a aussi des obligations sociales à rendre envers sa famille d'origine. Pour ne pas toujours demander l'accord de l'épouse, il résout certains problèmes de ses proches parents en coulisse. Il agit ainsi pour sa valorisation de soi. 


\section{Bibliographie}

Adepoju, Aderanti et Wariara Mbugua, "Les Mutations de la famille africaine ", dans Aderanti Adepoju (dir.), La Famille africaine. Politiques démographiques et développement, Paris, Karthala, coll. "Questions d'enfances", 1999, p. 59-84.

Ayimpam, Sylvie, "Violences sociales et exclusions réciproques en milieu africain. Le cycle des échanges de violence dans les rapports familiaux", dans Jacky Bouju et Mirjam de Bruijn (dir.), Violences sociales et exclusions. Le développement social de l'Afrique en question, Bulletin de l'APAD, n ${ }^{\text {os }}$ 27-28, 2005, p. 57-74.

Beaud, Stéphane et Florence Weber, Guide de l'enquête de terrain, Paris, La Découverte, 1998.

Bouju, Jacky, «La Sociabilité urbaine et la crise en Afrique de l'ouest : entre les défaillances de la solidarité familiale et la concurrence cérémonielle, on se cherche!", dans Georges Ravis-Giordani (dir.), Amitiés. Anthropologie et histoire, Aix-en-Provence, Publications de l'Université de Provence, 1999, p. 325-353.

Bozon, Michel et François de Singly, "Fortune et infortune de la femme mariée ", Population, 43e année, n 3, 1988, p. 683-685.

Caillé, Alain, Dé-penser l'économique, Paris, La Découverte/Mauss, 2005.

Cassel, Catherine, Victoria Bishop, Gillian Symon, Phil Johnson et Anna Buehring, "Learning to Be a Qualitative Management Researcher ", Management Learning, vol. 40, $\mathrm{n}^{\circ}$ 5, 2009, p. 513-532.

Glaser, Barney G., Theoretical Sensitivity, Mill Valley (CA), Sociology Press, 1978.

Godbout, Jacques et Johanne Charbonneau, Circulation du don dans la parenté, une roue qui tourne, Montréal, INRS-Urbanisation, 1996.

Godelier, Maurice, Les Métamorphoses de la parenté, Paris, Fayard, 2004.

Goffman, Erving, La Mise en scène de la vie quotidienne, Paris, Éditions de Minuit, 1973.

Leclercq, Hugues, «L'Économie populaire informelle de Kinshasa. Approche macro-économique ", Zaïre-Afrique, no 271, 1993, p. 17-36.

Marie, Alain, "Avatars de la dette communautaire. Crise des solidarités, sorcellerie et procès d'individualisation (itinéraires abidjanais) ", Alain Marie (dir.), L'Afrique des individus, Paris, Karthala, 1997, p. 249-328.

Marie, Alain, "Une anthropo-logique communautaire à l'épreuve de la mondialisation. De la relation de dette à la lutte sociale (l'exemple ivoirien) ", Cahiers d'études Africaines, n 166, 2002/2, p. 207-256. 
Mauss, Marcel, Sociologie et anthropologie, Paris, Presses universitaires de France, coll. "Quadrige ", 1985.

Mulumbwa Mutambwa, Georges et Jerry Kalonji wa Mpoyo, «L'économie morale : Approche lexicale », dans Pierre Petit (dir.), Lubumbashi 2000. La situation des ménages dans une économie de précarité, Lubumbashi, Observatoire du changement urbain, 2001, p. 166-176; http://www. congoforum.be/upldocs/Situation\%20menages\%20L\%20shi\%202000.pdf, site consulté en novembre 2013.

Muxel, Anne, Individu et mémoire familiale, $2^{\mathrm{e}}$ édition, Paris, Nathan, 2002.

Noret, Joël et Pierre Petit, Mort et dynamiques sociales au Katanga (République Démocratique du Congo), Paris, L'Harmattan, 2011.

Petit, Pierre (dir.), Ménages de Lubumbashi entre précarité et recomposition, Paris, L'Harmattan, 2003.

Pontault, Monique, Frères de sang, saurs de lait. Anthropologie d'une marginalisation familiale et sociale, Paris, L'Harmattan, 2001.

Sami Tchak, La Sexualité féminine en Afrique, Paris, L'Harmattan, 1999.

Sardan, Jean-Pierre Olivier de, La Rigueur du qualitatif. Les contraintes empiriques de l'interprétation socio-anthropologique, Louvain-la-neuve, Academia Bruylant, 2008.

Strauss, Anselm et Juliet Corbin, Basics of Qualitative Research: Grounded Theory Procedures and Techniques, Newbury Park (CA), Sage Publications, 1990.

Vangroenweghe, Daniel, Sida et sexualité en Afrique, Anvers, Éditions EPO, 2000.

Villers, Gauthier de, "Propositions introductives ", Phénomènes informels et dynamiques culturelles en Afrique, Cahiers Africains, $\mathrm{n}^{\text {os }} 19-20,1996$, p. 7-22. 